\title{
Design of optical matched filters
}

\author{
Pierre A. Humblet \\ Massachusetts Institute of Technology \\ Laboratory for Information and Decision Systems \\ Room 35-203 \\ Cambridge, Massachusetts 02139-4307 \\ Telephone: (617) 253-7250 Fax: (617) 258-8553*
}

\begin{abstract}
This paper studies the design and the performance of receivers using optical matched filters and direct detection, when the basic pulse shape consists a rectangular pulse of monochromatic light. The modulation format can be ASK, FSK or DPSK. Such receivers are useful when amplifier noise is the main source of uncertainty as they optimally reject additive white Gaussian noise. The performances of ASK and FSK receivers are reported, taking into account shot noise and the effects of post detection filtering.
\end{abstract}

"The anthors gatefully acknowledge support for this rescarch from NSE (grant NCR-8802991), and D) NRPA (grant F19628-90-C-0002) 


\section{Introduction}

In traditional receivers for optical fiber communication the shot noise and the electronic thermal noise are the main sources of uncertainty. The advent of optical amplifiers, which are expected to dramatically affect optical communication systems, profoundly changes the situation. An optical amplifier of power gain $G$ can be modelled as an ideal noiseless linear optical field amplifier together with a source of additive white Gaussian noise with twosided spectral density $(G-1) N_{s p} h \nu / 2$ per mode over the bandwidth of interest [14]. In that formula $N_{s p}$ is a coefficient greater than 1 (typically about 2) arising from imperfections in the amplifier, such as partial population inversion and losses, $h$ is Planck's constant and $\nu$ is the optical frequency. That additive noise often dominates the other sources of noise, both in direct reception receivers and in coherent systems where the received signal is mixed with that of a local oscillator. In the following discussion we assume that only the additive noise is present and recall some well known results of communication theory. They can be found in standard textbooks, e.g. [13], [12], [5],[6].

In the case of homodyne coherent reception (zero intermediate frequency) with known signal phase (synchronous detection) the effect of the additive noise can be optimally combatted by using an electronic filter matched to the envelope of the optical signals (a filter is matched to a signal if its proportional to the impulse response of the time reversed of the signal). The situation is similar in the case of heterodyne coherent reception (non zero intermediate frequency), with the proviso that a front end filter must be used to eliminate the additive noise in the image band. This filter must have a passband wide enough to pass the desired signal, and it must cutoff signals in the image band, two intermediate frequencies away. A $3 \mathrm{~dB}$ penalty is incurred with heterodyne detection if the front end filter is absent. The need for that filter can be avoided by using a quadrature receiver and rejecting the image band at the intermediate frequency. 
If the phase is random but constant during one symbol, then envelope (for heterodyne) or quadrature receivers (for homodyne or heterodyne) should be used.

For Amplitude Shift Keing (ASK, or ON/OFF) modulation, coherent synchronous receivers have a probability of error given by $Q\left(\sqrt{E / N_{0}}\right)$, where $E$ is the average energy of the signal at the receiver (half the $\mathrm{ON}$ energy), and $N_{0} / 2$ is the two-sided spectral density of the noise. The exact probability of error for envelope detection cannot be expressed easily, but it is about $\exp \left(-\left(1+E / N_{0}\right)\right)[2]$, which has the same asymptotic behavior as the previous expression. The penalty for using envelope detection is slight. For a probability of error of $10^{9}, E / N_{0}$ must be about 36 for synchronous detection, and 38.3 for envelope detection.

Note that in the context of optical amplifiers the ratio $E / N_{0}$ can be interpreted as the number of signal photons at the input of an ideal high gain amplifier that is the only source of noise in the system. The reader will recognize that these "number of photons" are similar to those usually quoted for heterodyne detection in absence of an optical amplifier. This is not surprising since the noises in both an ideal amplifier and an ideal heterodyne receiver are equivalent when referred to the input.

The probability of error for orthogonal binary Frequency Shift Keying (FSK) with synchronous detection is given by $Q\left(\sqrt{E / N_{0}}\right)$ ( $E$ is energy of each signal, thus also the average energy) and for envelope detection it is $.5 \exp \left(-E / 2 N_{0}\right)$. These schemes requires $E / N_{0}$ to be respectively 36 and 40 for a probability of error of $10^{-9}$.

For binary Phase Shift Keying (PSK) the probability of error is $Q\left(\sqrt{2 E / N_{0}}\right)$, i.e. $E / N_{0}$ equal to 18 for a probability of error of $10^{-9}$, twice as good as FSK. Of course synchronous detection is mandatory.

For binary Differential Phase Shift Keying (DPSK) (which only makes sense when synchronous detection is not possible), the probability of error given by $.5 \exp \left(-E / N_{0}\right)$, ignor- 
ing error propagation. $E / N_{0}$ needs to be only 20 for a probability of error of $10^{-9}$, which is only slightly more than for PSK.

Instead of using coherent receivers one can also employ bandpass matched filters followed by either synchronous or envelope detection. Their performances are identical to those of corresponding coherent receivers, synchronous or envelope. Our goal in this paper is to study the performance of direct detection receivers using optical bandpass match filters. They have some fundamental limitations.

Synchronous matched filter receivers must sample the output the filter with a timing precision measured in fraction of the period of the carrier. This is not possible at optical frequencies because detectors respond only to the envelope of the signal. Thus we can only hope to do as well as envelope detectors. Another problem with optical detection is that few photons will be collected during the ideal infinitesimal sampling interval called for by the classical theory. To prevent the shot noise from becoming dominant it is always necessary to integrate the output of the matched filter for some time, thus degrading its performance.

The main contribution of this paper is to design optical filters that are almost perfectly matched to rectangular pulses of monochromatic light, and which could be used in ASK, FSK and DPSK systems when chirping and phase noise are not significant. We also report on the penalty that is caused by the shot noise and by integrating the output of the matched filter. Before attacking those topics we make a few more comparisons between coherent and direct detection systems.

Many optical sources have significant linewidth and the signal phase changes during the transmission of one symbol. Optical filters will be affected by phase noise exactly like coherent receivers with envelope detection. The absence of a local oscillator typically reduces the effective intensity of the phase noise by $50 \%$ for optical filters, compared to coherent receivers. To the contrary, frequency stabilization is probably more critical for 
optical filters, because they cannot readily track frequency fluctuations in the transmitter. The previous discussion has implicitly assumed that the signal was polarized and that the other polarization was filtered out. Allowing both polarizations decreases the performances somewhat, but not nearly as much as a polarization mismatch degrades coherent receivers.

\section{Optical matched filters}

Suppose we have a rectangular pulse $s(t)$ of monochromatic light at frequency $\nu$, with length $T$ and energy $E: s(t)=\sqrt{2 E / T} \cos (2 \pi \nu t), t \in[0, T]$, as in figure 2.a. For mathematical convenience we will first assume that $\nu=2^{k} / T$ for some moderately large integer $k$. Assume one divides the signal equally in two branches, which are recombined after undergoing a differential delay of exactly $T / 2$. The envelope of the recombined field would then be as in figure 2.b. It has length $1.5 T$, with 2 up steps and 2 down steps.

The device that would realize what we have just described is simply a Mach-Zehnder filter with differential delay $T / 2$. In figure 1 we use the ouput diagonally opposed to the input so as to produce the sum of the input signal and its delayed version. The other output branch produces the difference.

If the output signal is split again and recombined after a differential delay of $T / 4$, the envelope will be as in figure 2.c. Note that there are 4 up steps and 4 down steps and the length is now $1.75 T$. If one use $k$ stages, using differential delays that decrease by a factor of 2 every time, the recombined field will have $2^{k}$ up steps, as many down steps, and a length $\left(2-2^{-k}\right) T$. Note that the amplitude at the center is constant in all these figures. One sees that the output converges geometrically to the triangle of base $2 T$ that would be produced by a true matched filter (figure 2.d). If $\nu \neq 2^{k} T$ then the delays must be adjusted around their nominal value so that the signals add in phase at the Mach-Zehnder outputs.

The process can also be visualized in the frequency domain. The $k$ stage filter has a 
frequency response given by $\prod_{i=1}^{k} \cos \left(2^{-k} \pi f T\right)=\sin (\pi f T) / 2^{k} \sin \left(2^{-k} \pi f T\right)$. This is displayed in figure 3 for $k=3$, together with the response $\sin (\pi f T) / \pi f T$ of a matched filter (of course those responses are actually centered at $\pm \nu$ ). Note that the approximate filter is close to ideal for frequencies smaller than $1 / T$ (where the signal energy lies), but its response magnitude is periodic with period $2^{k} / T$, thus letting in the noise in the undesired periods. In practice our filter would be cascaded with another optical bandpass filter of bandwidth $2^{k} / T$, thus eliminating the undesired noise and smoothing the output signal. The other filter could be a grating, or a thin film interference filter possibly grown directly on the facet of a fiber, as is commonly done in fiber Fabry-Perot interferometers [7], [8]. For example if $T$ is $1 \mathrm{~ns}$ and $k$ is 7 , the bandwith of the other filter would be about $1 \mathrm{~nm}$ if $\nu$ is in the $1.5 \mu \mathrm{m}$ band.

The filter just described has been proposed before as a channel selection filter for optical frequency division multiaccess systems [10]. Its optimal noise rejection property has apparently not been pointed out. Integrated versions of the filter have been realized [3], [9], [11] using silica on silicon technologies. One implementation had $k=7, T=.2 \mathrm{~ns}$ and an insertion loss of about $6 \mathrm{~dB}$. Such a loss affects the additive noise as well as the signal and thus it is not very significant when the additive noise is the main source of uncertainty. The next section will provide more quantitative statements.

The previous filter can be used alone with ASK modulation together with a single photodiode. After filtering, the output current must be compared to a threshold to arrive at a decision (see figure 4.a).

In the case of binary FSK modulation, 2 matched filters would be needed, one for each tone, together with a balanced receiver consisting of two photodiodes as in figure 4.b. Decisions can be based on the sign of the current, no energy dependent threshold is necessary. 
In the case of DPSK we can view the signal as consisting of two parts: a reference part (actually the previous bit) followed by a signal part, which is either the reference signal delayed by $T$ or its negative. The filter matched to one signal should thus have response $s(-t)+s(-t+T)$, while the other filter should have response $s(-t)-s(-t+T)$. Of course the implementation of those operations is very simple: the matched filter $s(-t)$ for an isolated pulse should be followed by a Mach-Zehnder filter with differential delay $T$ (figure 4.c). One output branch has impulse response $.5(\delta(t)-\delta(t-T))$ while the other branch has response $.5(\delta(t)+\delta(t-T))$, so that the cascade has the desired response. The resulting structure is simply a matched filter for pulses of length $2 T$, with two outputs being used! Again a balanced photoreceiver is called for.

In the next section we focus on some fundamental issues for ASK and FSK: the effect of post filtering and of shot noise. More complete studies will be reported elsewhere.

\section{Shot noise and post filtering}

As already indicated shot noise imposes fundamental limitations on optical systems. In particular the output of the matched filter cannot be sampled using a photodetector, but to the contrary the output from the detector must be low pass filtered. This causes some deviation from the classical theory. We have performed an analysis, too long to be reported here, of the degradation. We assume that the low pass filter is an ideal integrate and dump filter, with an integration time from $-\alpha T$ to $\alpha T$. Thus a small $\alpha$ corresponds to weak filtering. $\alpha$ cannot be larger than .5 without causing too much intersymbol interference.

For binary FSK with wide frequency deviation we plot in figure 5 the necessary $E / N_{0}$ for $P_{e}=10^{-9}$ as a function of $\alpha$ for various values of $N_{0} . N_{0}$ is expressed in photons. For example if the only source of noise is an ideal optical amplifier with $G=101$ placed just before the detector, then $N_{0}=100$. If there is a $6 \mathrm{~dB}$ loss between the amplifier and the 
detector then $N_{0}$ decreases to 25 .

For low $\alpha$ the increase in required $E / N_{0}$ is due to the shot noise. At larger $\alpha$ the penalty comes from the fact that the collected signal energy increases more slowly than the average noise energy. This graph does not take the thermal noise into account. We expect it to cause the same general effect as the shot noise, but with a different magnitude. Similarly the effect of intersymbol interference will magnify the penalty for using a large $\alpha$.

We expect small deviation FSK to behave in the same way for small $\alpha$, but its performance for larger $\alpha$ might be somewhat different. Similarly DPSK should follow the FSK curve, except that it requires only half as many photons.

Results appear in figure 6 for ASK modulation. threshold, and to optimize on the threshold. The required signal

\section{Conclusion}

This paper has outlined the structure of optical filters matched to pulses of monochromatic waves. They could be used with ASK, FSK and DPSK modulations in systems where chirp and phase noise are negligible, and where the main source of uncertainty is spontaneous amplifier noise. For wide deviation FSK and ASK we have shown the effects of the unavoidable penalty arising in direct detection optical systems from shot noise and from the need to integrate the output of the photodetector. These effects are small.

\section{References}

[1] R.M.Gagliardi and S. Karp, Optical Communications, Wiley 1976.

[2] P.A. Humblet and M. Azizoglu, "On the Bit Error Rate of Lightwave Systems with Optical Amplifiers", MIT Report LIDS-P-2015, submitted for publication, 1991. 
[3] K. Inoue et al., "A Four-Channel Optical Waveguide Multi/Demultiplexer Fabricated for $5 \mathrm{GHz}$ Spaced Optical FDM Transmission", J. Lightwave Technol., Vol. 6, No.6, pp. 339-345 (Jun. 1988).

[4] T. Kailath, Some Integral Equations with "Nonrational" Kernels, IEEE Trans. on Inform. Theory, Vol. IT-12, No. 4, Oct. 66 , pp. 442-447

[5] J. G. Proakis, Digital Communications, McGraw-Hill, 1983.

[6] M. Schwartz, Information Transmission, Modulation and Noise, McGraw-Hill, 1990

[7] J. Stone, "Optical-fibre Fabry-Perot Interferometer with a Finesse of 300", Electr. Lett., Vol. 21, pp. 504-505, (1985).

[8] J. Stone and L.W. Stultz, "Pigtailed High-Finesse Tunable Fibre Fabry-Perot Interferometers with Large, Medium and Small Free Spectral Ranges", Electr. Lett., Vol. 23, No. 15 , pp. 781-782, (Jul. 1987).

[9] B.H. Verbeek et al., "Integrated Four-Channel Mach-Zehnder Multi/Demultiplexer Fabricated with Phosphorous Doped $\mathrm{SiO}_{2}$ waveguides on $\mathrm{Si}$, J. Lightwave Technol., Vol. 6, No.6, pp. 1011-1015 (Jun. 1988).

[10] H. Toba et al., "A Conceptional Design on Frequency-Division-Multiplexing Distribution with Optical Tunable Filters", IEEE J. Select. Areas in Comm. Vol. 4, pp. 686-694 (1986).

[11] N. Takato et al., "Silica-Based Integrated Optic Mach-Zehnder Multi/Demultiplexer Family with Channel Sopacing of 0.01-250 nm", IEEE J. Select. Areas in Comm. Vol. 8, No. 6, pp. 1120-1127, (Aug. 1990).

[12] H.L. VanTrees, Detection, Estimation, and Modulation Theory, Part I, Wiley 1968. 
[13] J.M. Wozencraft and I.M. Jacobs, Principles of Communication Engineering, Wiley 1965.

[14] A. Yariv, Optical Electronics, Holt, Rinehart and Winston, 1985. 

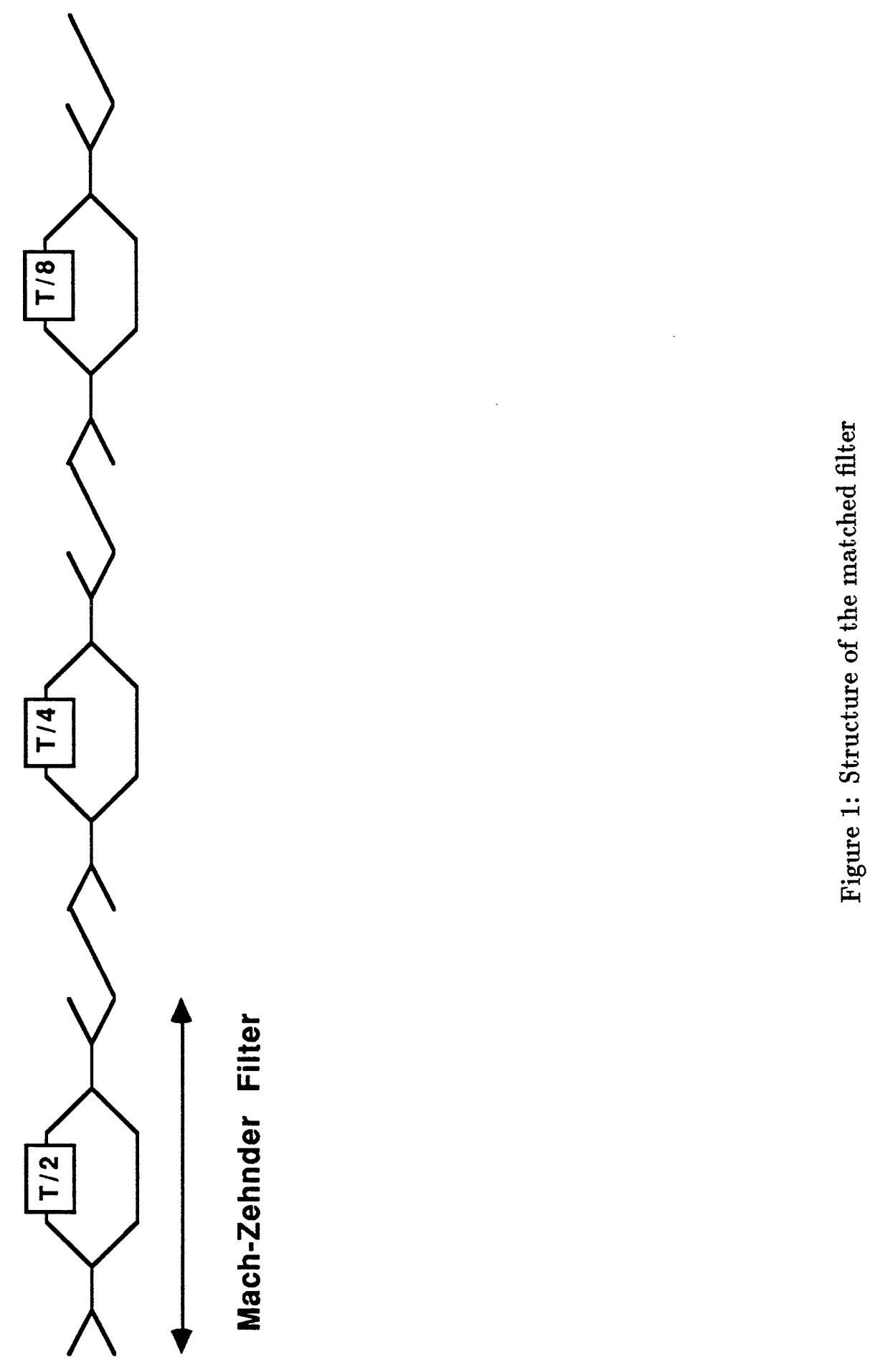


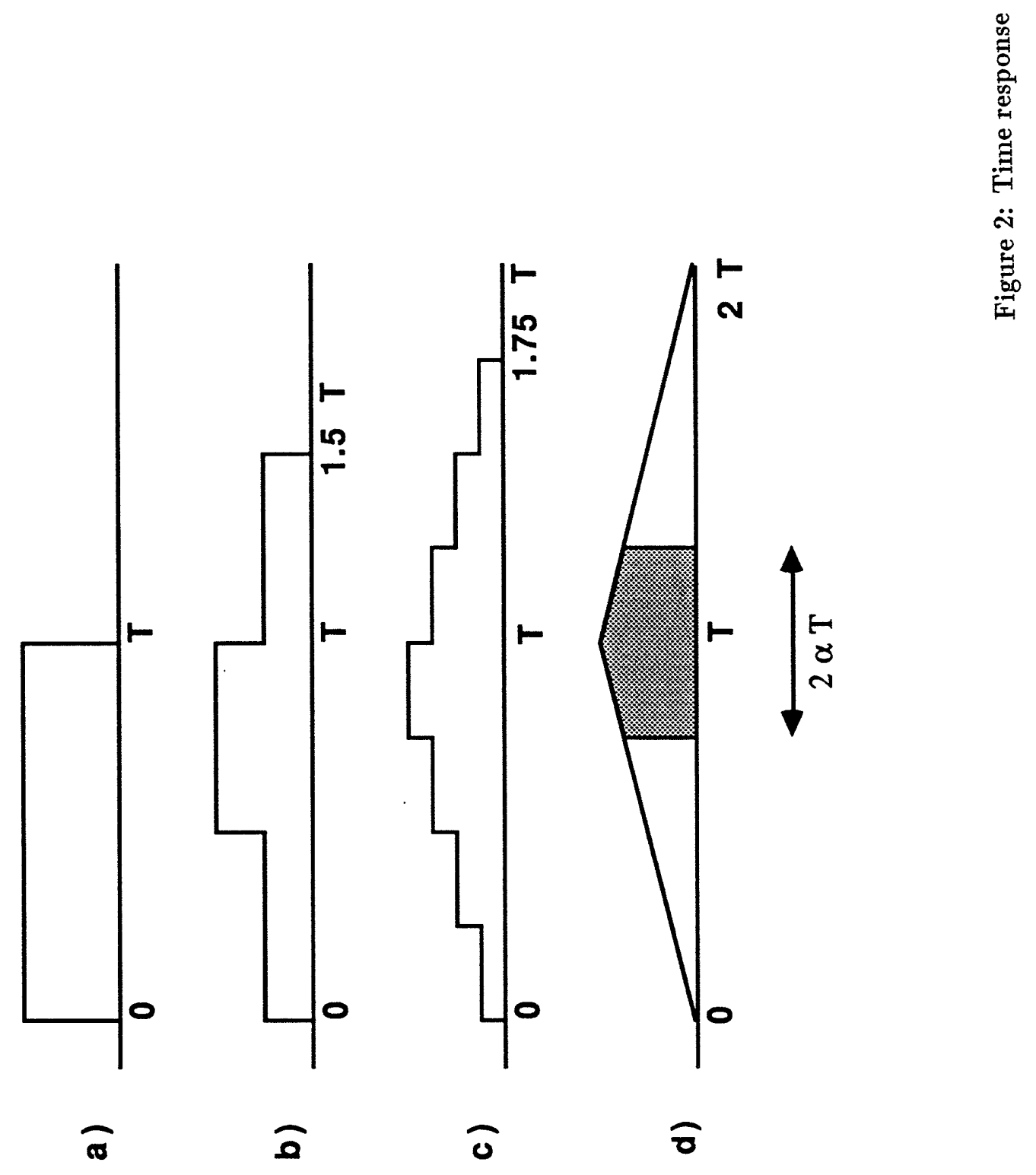




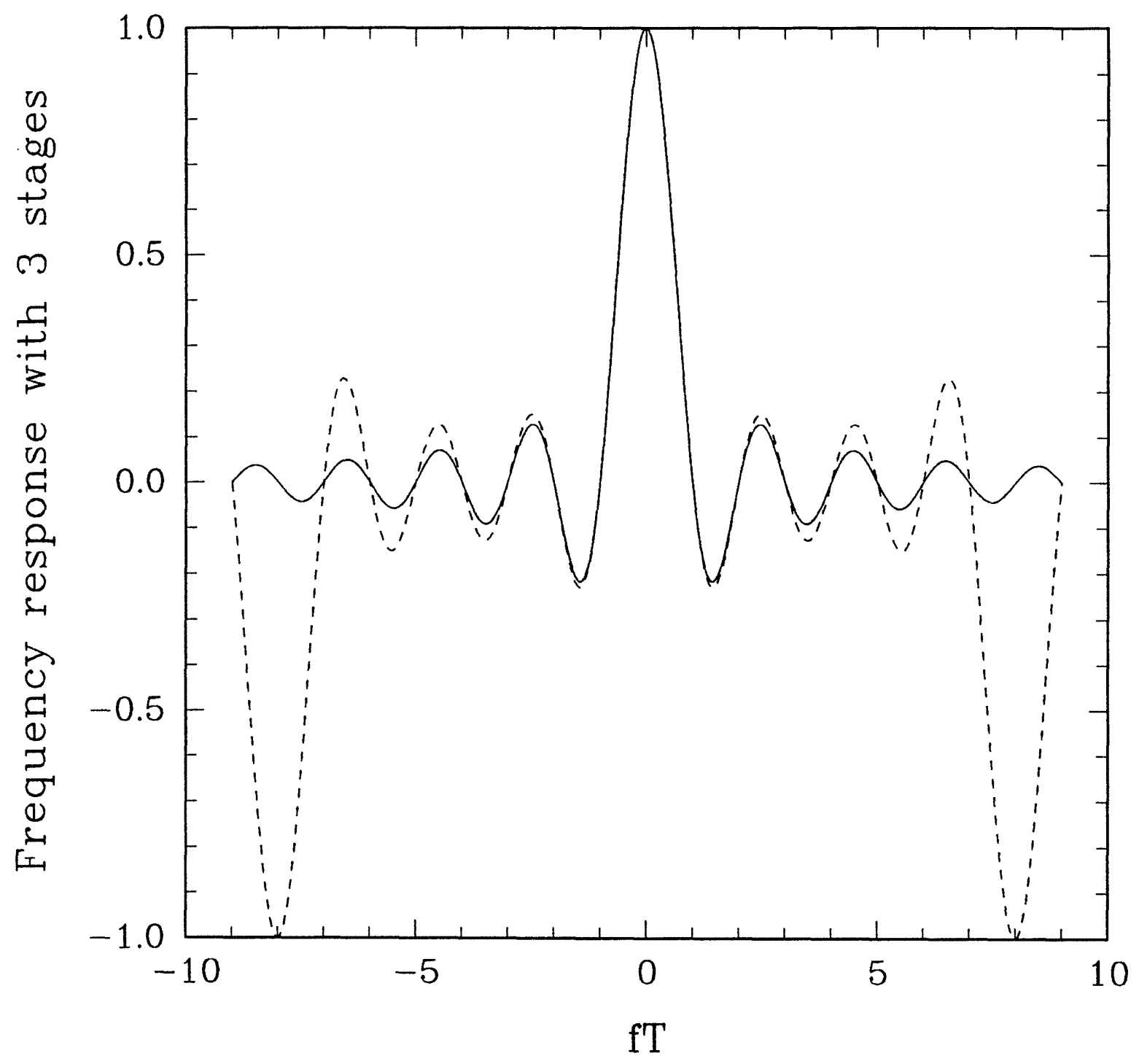

Figure 3: Frequency response 


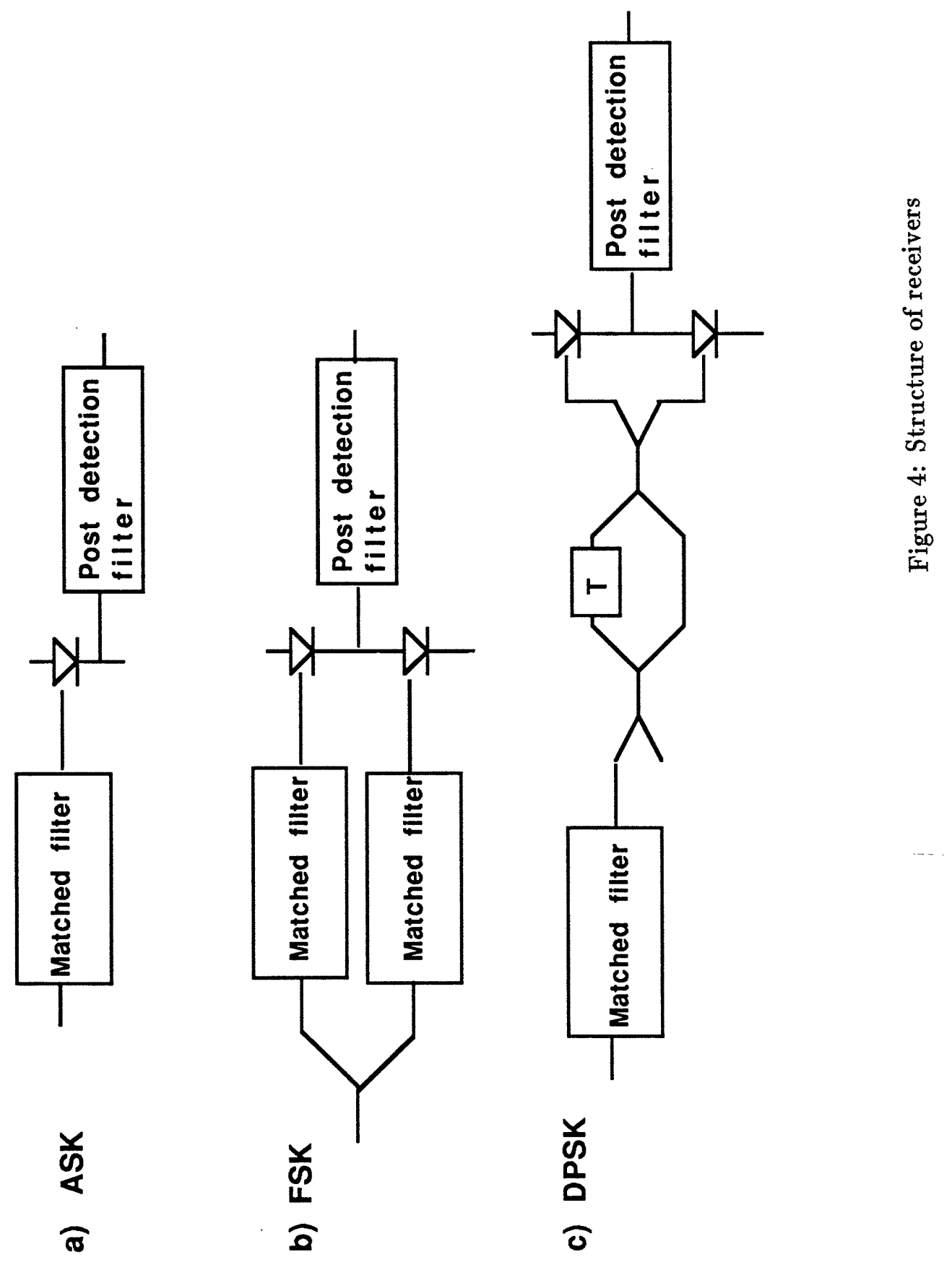




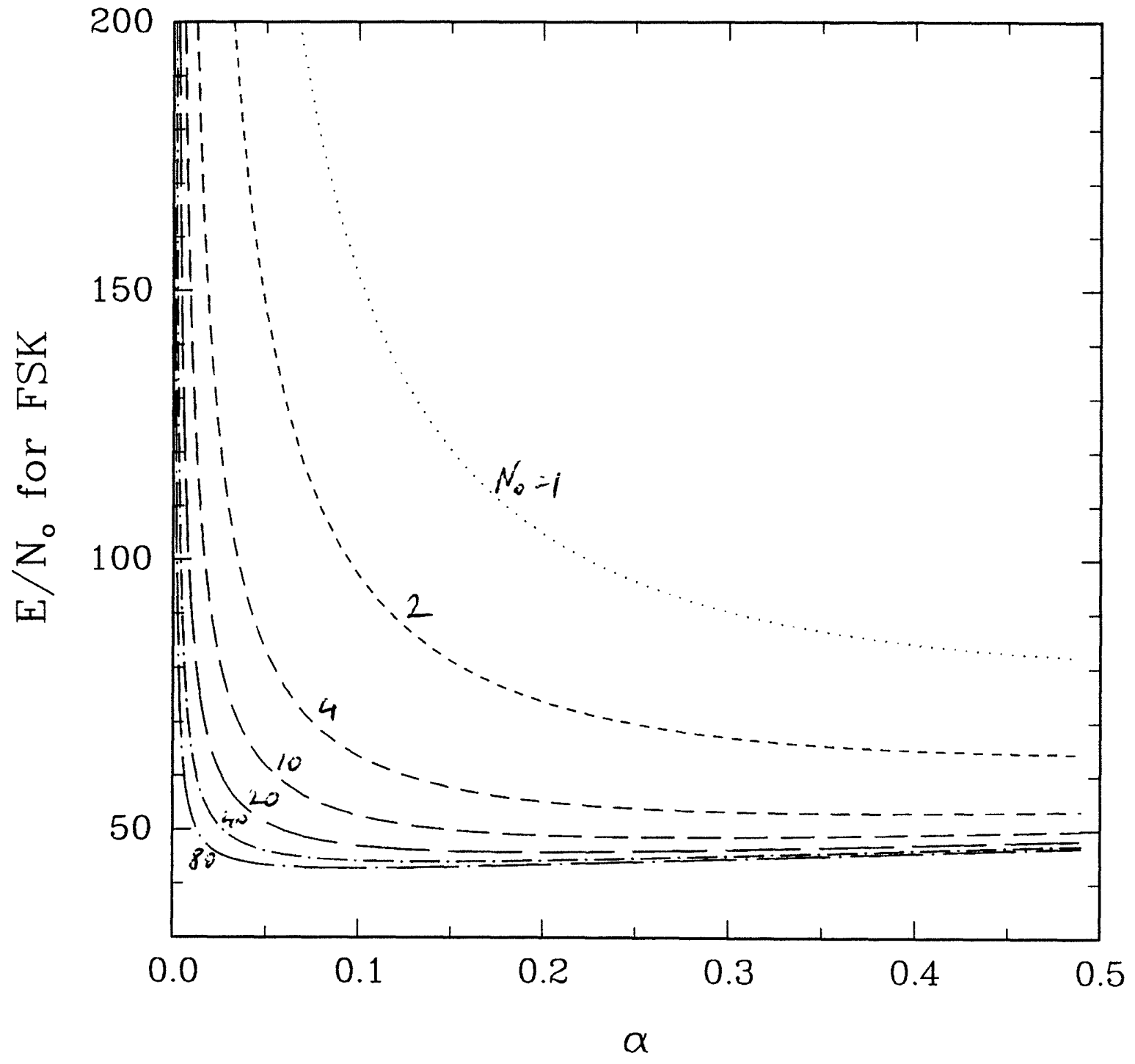

Figure 5: Signal to noise ratio for FSK, $P_{e}=10^{-9}$ 


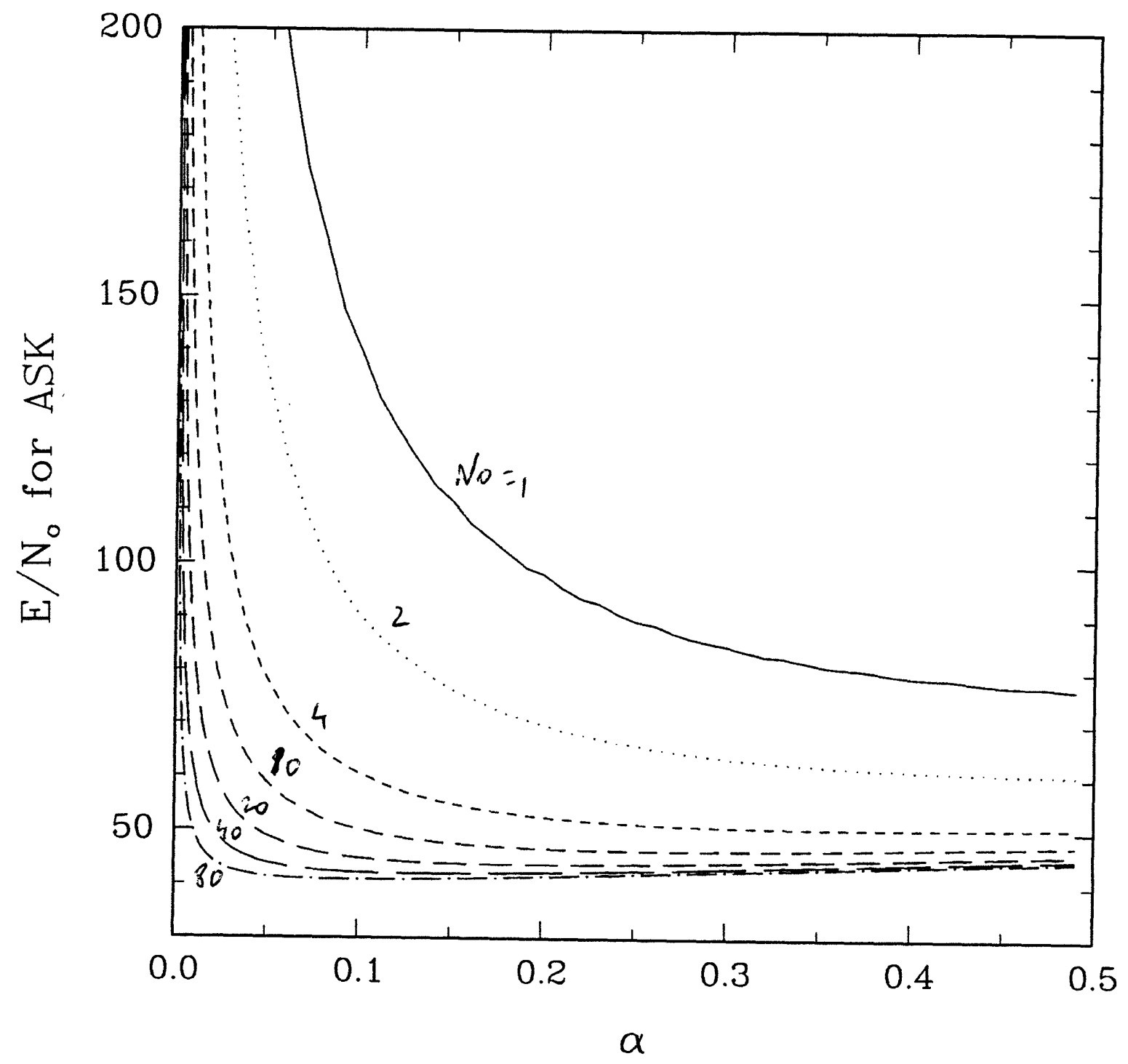

Figure 6: Signal to noise ratio for $\mathrm{ASK}, P_{e}=10^{-9}$ 